\title{
Relative uncertainty aversion and additively representable set rankings*
}

\author{
WALTER BOSSERT \\ Département de Sciences Economiques and CIREQ, Université de Montréal \\ walter. bossert@umontreal . ca \\ ARKADII SLINKO \\ Department of Mathematics, University of Auckland \\ a.slinko@auckland.ac.nz
}

August 2004; revised January 2005

\begin{abstract}
This paper proposes a definition of relative uncertainty aversion for decision models under complete uncertainty. It is shown that, for a large class of decision rules characterized by a set of plausible axioms, the new criterion yields a complete ranking of those rules with respect to the relative degree of uncertainty aversion they represent. In addition, we address a combinatorial question that arises in this context, and we examine conditions for the additive representability of our rules. Journal of Economic Literature Classification No.: D81.
\end{abstract}

Keywords: Choice under complete uncertainty, uncertainty aversion, additive representability.

* We thank Nick Baigent, Luis Corchón, Ínigo Iturbe-Ormaetxe, Vincent Merlin, Maurice Salles, Horst Zank and several seminar audiences for comments and suggestions. Financial support from the Social Sciences and Humanities Research Council of Canada (SSHRCC) and the New Zealand Institute of Mathematics and its Applications (NZIMA) is gratefully acknowledged. 


\section{Introduction}

Decision rules for choice under complete uncertainty rank actions on the basis of the sets of possible outcomes they induce. The term complete uncertainty is intended to describe a situation where an agent knows the set of possible consequences of an action but cannot assign probabilities to these outcomes. The choice of an action is assumed to proceed in two steps. First, a ranking of sets of possible outcomes is established and then the agent selects an available action that is associated with a set of consequences that is best according to the ranking. In the model considered here, the universal set of outcomes is non-empty and finite and the ranking of sets to be established is an ordering. Consequently, best elements always exist and the above-described procedure is well-defined. Rankings of sets that are interpreted as sets of possible outcomes under complete uncertainty have been analyzed in numerous axiomatic studies such as the pioneering contribution of Kannai and Peleg (1984); see also Bandyopadhyay (1988), Barberà, Barrett and Pattanaik (1984), Barberà and Pattanaik (1984), Bossert (1989), Fishburn (1984), Heiner and Packard (1984), Holzman (1984a,b), Nitzan and Pattanaik (1984) and Pattanaik and Peleg (1984). A survey can be found in Barberà, Bossert and Pattanaik (2004).

Clearly, there are approaches to choice under complete uncertainty other than the set-based model analyzed here. For example, Arrow and Hurwicz (1972), Barrett and Pattanaik (1993), Cohen and Jaffray (1980), Luce and Raiffa (1957), Maskin (1979) and Milnor (1954) model non-probabilistic choice under uncertainty by specifying a set of possible states of the world and an outcome results from combining a feasible action with a possible state. In this more conventional setting, actions are ranked by comparing the vectors of contingent outcomes that are generated by this approach and, in contrast to our set-based model, it is possible to keep track not only of the set of possible outcomes but also, for example, of the number of states in which a given outcome materializes. In defense of the set-based model, however, it should be noted that we may want to avoid attaching importance to the number of states where the same outcome materializes because this number may be the result of an arbitrary subdivision of otherwise indistinguishable states. In addition, if the number of possible states of the world is large, an agent may find it too complex to take into account the entire vector of contingent outcomes. Restricting attention to the set of possible outcomes is one way of arriving at a more tractable representation of the information that is available. Finally, in a Rawlsian veil-of-ignorance framework (see Rawls, 1971), it may not be obvious how states and outcomes can be distinguished in a satisfactory manner and, again, the set-based model seems very suitable 
in these circumstances. We do, of course, not claim that the set-based approach represents the most suitable model of choice under complete uncertainty in all circumstances, but the above discussion demonstrates that there are compelling arguments in favor of adopting it in numerous situations. Thus, analyzing the properties of set-based decision rules is an important task.

One important feature of a decision rule under uncertainty is the degree of risk aversion or uncertainty aversion it represents. Attitudes such as risk aversion and uncertainty aversion have been analyzed thoroughly in the linear expected-utility framework of von Neumann and Morgenstern (1944, 1947); see, for example, Arrow (1971) and Pratt (1964). Chew, Karni and Safra (1987) examine risk and uncertainty aversion in the presence of rank-dependent probabilities, and Wakker (1990) characterizes optimism and pessimism in non-linear probability models. A definition of uncertainty aversion for Choquet expectedutility models is presented in Schmeidler (1989), and Epstein (1999) formulates a notion of uncertainty aversion that is particularly suitable in a Savage (1954) framework. Bossert (1997) provides a definition of uncertainty aversion for the set-based model. However, his definition is an absolute definition in that it only allows us to establish whether or not a given ordering of sets of possible outcomes is uncertainty averse in a specific sense - it does not permit comparisons of different decision rules with respect to their relative degree of uncertainty aversion.

In this paper, we suggest an intuitively appealing definition of relative uncertainty aversion that allows us to rank all set-based decision rules within a given class with respect to the uncertainty aversion they represent. Although a formal definition will have to wait until we have introduced some basic notation and definitions, a simple example serves to introduce the basic idea. Suppose we have four objects numbered 1 to 4 where 1 is best, 2 is second-best, followed by 3 , and 4 is worst. Suppose one ordering of sets of possible outcomes declares both the set $\{2\}$ and the set $\{3\}$ better than the set $\{1,4\}$ whereas, according to a second ordering, $\{1,4\}$ is better than $\{3\}$ but $\{2\}$ is better than $\{1,4\}$. Abstracting from all other comparisons for the moment, it is plausible to say that the first ranking exhibits more uncertainty aversion than the second: an agent with the second ranking is willing to forgo the certain outcome 3 in favor of the possibility of getting the best choice 1, even though the worst possible outcome 4 is also a possibility. Clearly, this is not the case for the first ranking. Our criterion for comparing orderings of sets with respect to the uncertainty aversion they represent is based on this intuition. Loosely speaking, the closer a certain outcome has to be to the better of two possible outcomes in order to be declared at least as good, the more uncertainty aversion the ranking represents. 
Clearly, without any restrictions on the set rankings, it cannot be expected that such a criterion can be applied consistently to all comparisons. Interestingly, it turns out that three plausible axioms restrict the class of possible orderings in a way such that the proposed criterion generates an uncertainty-aversion ordering defined on this class.

In Section 2, we introduce two fundamental axioms and characterize all set ranking rules that satisfy them. A new translation-neutrality axiom is defined in Section 3 and we show that the rules characterized by it and the previous axioms can be given a convenient diagrammatic representation. Section 4 discusses our new definition of relative uncertainty aversion if applied to the orderings characterized in Section 3. Furthermore, we prove an asymptotic combinatorial result regarding the number of orderings in our class. Section 5 examines the issue of additive representability and we show that some, but not all, of our orderings are additively representable in a weak sense. Section 6 concludes.

\section{Set rankings}

Our goal is to examine rankings of all non-empty subsets of a finite and non-empty universal set of objects, based on a ranking of the objects themselves. We interpret these sets as sets of possible outcomes and their ranking as a decision rule in the context of choice under complete uncertainty. We assume that the objects are ranked by an antisymmetric ordering, where an ordering is a reflexive, transitive and complete binary relation. Without loss of generality, we simplify notation by identifying the objects with natural numbers ordered from best to worst, that is, the universal set is given by $[n]=$ $\{1, \ldots, n\}$ with $n \in \mathbb{N}$ and the antisymmetric ordering on $[n]$ is such that, for all $i, j \in[n]$, $i$ is at least as good as $j$ if and only if $i \leq j . \mathcal{P}[n]$ is the set of all non-empty subsets of

$[n]$. For $A \in \mathcal{P}[n],|A| \in \mathbb{N}$ is the cardinality of $A$. We label the elements in $A$ such that $A=\left\{a_{1}, \ldots, a_{|A|}\right\}$ with $a_{1}<\ldots<a_{|A|}$ if $|A| \geq 2$ and $A=\left\{a_{1}\right\}=\left\{a_{|A|}\right\}$ if $|A|=1$. Thus, for any $A \in \mathcal{P}[n]$, the best element in $A$ is $a_{1}$ and the worst element in $A$ is $a_{|A|}$.

$R$ is an ordering on $\mathcal{P}[n]$ and its asymmetric and symmetric parts are denoted by $P$ and $I$. Kannai and Peleg (1984) show that some plausible properties imposed on the ordering $R$ imply that any set $A \in \mathcal{P}[n]$ must be indifferent to the set consisting of the best element and the worst element in $A$. Related results appear, for instance, in Barberà, Barrett and Pattanaik (1984) and in Bossert, Pattanaik and Xu (2000); see also Arrow and Hurwicz (1972) for an analogous implication in a more traditional decision-theoretic context. Nehring and Puppe (1996) characterize all rankings that depend on best and worst elements only in a model where the universal set is uncountable and endowed with 
some topological structure. In this paper, we weaken one of the Kannai-Peleg axioms because their properties result in an impossibility.

Our first axiom is a dominance property which requires that the addition of an object that is better (worse) than all objects in a given set $A \in \mathcal{P}[n]$ leads to a set that is better (worse) than $A$.

Dominance. For all $A \in \mathcal{P}[n]$ and for all $i \in[n]$,

(i) if $i<j$ for all $j \in A$, then $A \cup\{i\} P A$;

(ii) if $i>j$ for all $j \in A$, then $A P A \cup\{i\}$.

In recognition of the use of this axiom in Gärdenfors (1976), dominance is referred to as the Gärdenfors principle in Kannai and Peleg (1984). See also Kim and Roush (1980) for a discussion.

The next axiom is a weakening of Kannai and Peleg's (1984) independence condition (which they call monotonicity). Their axiom requires that if there exists a strict preference between two sets $A$ and $B$, adding the same alternative to both sets does not reverse this strict ranking. We weaken this requirement considerably by limiting the scope of its conclusion to cases where the worst (best) element is the same in both sets and the object to be added to both sets is better (worse) than all elements in the union of the two sets. By considering additions of better (worse) elements only and by restricting attention to additions where the two initial sets have a common worst (best) element, we weaken the Kannai-Peleg axiom in a way that is consistent with the idea of reducing the complexity involved in a decision problem. Thus, this weakening is in line with one of the features of the set-based model alluded to in the introduction, namely, the reduction in complexity when assessing changes in the sets of possible outcomes. Moreover, our version of the axiom can be shown to be, together with the dominance axiom, a minimal requirement (in a sense made precise in Theorem 2 below) to obtain the result that any set is indifferent to the set consisting of its best element and its worst element.

Conditional independence. For all $A, B \in \mathcal{P}[n]$ and for all $i \in[n]$,

(i) if $A P B, a_{|A|}=b_{|B|}$ and $i<j$ for all $j \in A \cup B$, then $A \cup\{i\} R B \cup\{i\}$;

(ii) if $A P B, a_{1}=b_{1}$ and $i>j$ for all $j \in A \cup B$, then $A \cup\{i\} R B \cup\{i\}$.

Dominance and conditional independence are natural axioms to impose if $R$ is interpreted as a ranking of sets of possible outcomes under complete uncertainty. 
The following theorem strengthens one of Kannai and Peleg's (1984) results by weakening their independence axiom to conditional independence. As noted in Bossert (1989), the transitivity of $R$ assumed in the Kannai-Peleg result can be weakened to transitivity of the asymmetric part $P$ of $R$. This is the case for our formulation as well.

Theorem 1. If an ordering $R$ on $\mathcal{P}[n]$ satisfies dominance and conditional independence, then $A I\left\{a_{1}, a_{|A|}\right\}$ for all $A \in \mathcal{P}[n]$.

Proof. Let $A \in \mathcal{P}[n]$. If $A$ contains less than three elements, the claim follows immediately from the reflexivity of $R$. Now suppose $|A| \geq 3$. By repeated application of part (i) of dominance and transitivity, it follows that $\left\{a_{2}, \ldots, a_{|A|}\right\} P\left\{a_{|A|}\right\}$ and, using part (i) of conditional independence, we obtain

$$
A R\left\{a_{1}, a_{|A|}\right\}
$$

Repeated application of part (ii) of dominance and transitivity yields $\left\{a_{1}\right\} P\left\{a_{1}, \ldots, a_{|A|-1}\right\}$, and part (ii) of conditional independence implies

$$
\left\{a_{1}, a_{|A|}\right\} R A
$$

Combining (1) and (2), we obtain $A I\left\{a_{1}, a_{|A|}\right\}$.

As is clear from the proof of this theorem, its conclusion is valid not only for orderings but for any reflexive relation whose asymmetric part is transitive.

Theorem 1 can be used to characterize all orderings satisfying our two axioms. It reduces an ordering satisfying dominance and conditional independence to the ordering of one-element and two-element subsets satisfying the same properties. We identify twoelement subsets of $[n]$ with the elements of the set of all pairs $\{(i, j) \mid i<j\}$. Furthermore, we identify a singleton $\{i\}$ with the pair $(i, i)$. Thus, all one-element and two-element subsets of $[n]$ are identified with the elements of the set

$$
\mathcal{S}[n]=\{(i, j) \mid i, j \in[n] \text { and } i \leq j\}
$$

An ordering on $\mathcal{S}[n]$ is denoted by $\succeq$ with asymmetric and symmetric parts $\succ$ and $\sim$. As shown in the following theorem, an ordering on $\mathcal{P}[n]$ satisfies dominance and conditional independence if and only if there exists a corresponding ordering on $\mathcal{S}[n]$ (in the sense that will be explained below) which possesses the following monotonicity property. 
Monotonicity. For all $i, j, k \in[n]$,

(i) if $i<j \leq k$, then $(i, k) \succ(j, k)$;

(ii) if $i \leq j<k$, then $(i, j) \succ(i, k)$.

The following theorem characterizes all orderings on $\mathcal{P}[n]$ satisfying dominance and conditional independence.

Theorem 2. An ordering $R$ on $\mathcal{P}[n]$ satisfies dominance and conditional independence if and only if there exists a monotonic ordering $\succeq$ on $\mathcal{S}[n]$ such that, for all $A, B \in \mathcal{P}[n]$,

$$
A R B \Leftrightarrow\left(a_{1}, a_{|A|}\right) \succeq\left(b_{1}, b_{|B|}\right)
$$

Proof. It is straightforward to verify that if there exists a monotonic ordering $\succeq$ on $\mathcal{S}[n]$ such that (3) is satisfied, then $R$ satisfies dominance and conditional independence. Conversely, suppose $R$ satisfies the two axioms. By Theorem 1 and because $R$ is an ordering, $\succeq$ is a well-defined ordering satisfying (3). To show that $\succeq$ satisfies part (i) of monotonicity, suppose $i, j, k \in[n]$ are such that $i<j \leq k$. If $j=k,(i, k) \succ(j, k)$ follows from part (i) of dominance. If $j<k$, Theorem 1 implies $\{i, k\} I\{i, j, k\}$ and part (i) of dominance implies $\{i, j, k\} P\{j, k\}$. By transitivity, $\{i, k\} P\{j, k\}$ and, by (3), $(i, k) \succ(j, k)$. The proof of part (ii) is analogous.

\section{Translation neutrality}

As established in the previous section, the set of orderings on $\mathcal{P}[n]$ that satisfy dominance and conditional independence is quite diverse. To introduce a class of orderings for which relative uncertainty aversion may be defined in an unambiguous manner, we have to narrow down this class. We do so by introducing another axiom which is similar in spirit to the neutrality axiom familiar from the literature. In our definition of relative uncertainty aversion, we make use of the relative differences in position of the objects and, as a consequence, the classical neutrality axiom is not suitable. Indeed, neutrality requires that the relation $R$ is insensitive with respect to changes in two sets $A$ and $B$ that leave the relative rankings of all elements in $A \cup B$ unchanged (see, for instance, Barberà, Bossert and Pattanaik, 2004, for a formal definition and discussion). This rules out any utilization of the relative differences in position of the objects. Instead of neutrality, 
we introduce an alternative axiom that allows relative differences between positions to matter. Our axiom requires that if the best (worst) elements of two sets are translated by one position, the relative ranking of the resulting sets is the same as that of the originals.

For $A \in \mathcal{P}[n]$ with $a_{1} \neq 1$, define

$$
A^{-}= \begin{cases}\left\{a_{1}-1, a_{1}\right\} & \text { if }|A|=1 \\ \left\{a_{1}-1, a_{2}, \ldots, a_{|A|}\right\} & \text { if }|A| \geq 2\end{cases}
$$

and, for $A \in \mathcal{P}[n]$ with $a_{|A|} \neq n$, define

$$
A^{+}= \begin{cases}\left\{a_{|A|}, a_{|A|}+1\right\} & \text { if }|A|=1 \\ \left\{a_{1}, \ldots, a_{|A|-1}, a_{|A|}+1\right\} & \text { if }|A| \geq 2 .\end{cases}
$$

Our translation-neutrality axiom is defined as follows.

Translation neutrality. For all $A, B, C, D \in \mathcal{P}[n]$,

(i) if $C=A^{-}$and $D=B^{-}$, then $A R B \Leftrightarrow C R D$;

(ii) if $C=A^{+}$and $D=B^{+}$, then $A R B \Leftrightarrow C R D$.

To illustrate how this axiom differs from the usual neutrality condition, consider the following example. Suppose that $n=5$ and $\{2,5\} P\{4\}$. Translation neutrality and neutrality both require $\{1,4\} P\{3\}$. Neutrality also demands, for example, $\{1,3\} P\{2\}$ whereas translation neutrality does not. Conversely, translation neutrality requires, in addition, $\{1,5\} P\{3,4\}$ but neutrality does not.

Given the axioms of Theorem 2, it is easily seen that translation neutrality of $R$ on $\mathcal{P}[n]$ is equivalent to the following property of $\succeq$ on $\mathcal{S}[n]$. The choice of terminology for this axiom will become clear after introducing a geometric interpretation.

Parallel-displacement invariance. For all $(i, j),(k, \ell) \in \mathcal{S}[n]$,

(i) if $(i-1, j),(k-1, \ell) \in \mathcal{S}[n]$, then $(i, j) \succeq(k, \ell) \Leftrightarrow(i-1, j) \succeq(k-1, \ell)$;

(ii) if $(i, j+1),(k, \ell+1) \in \mathcal{S}[n]$, then $(i, j) \succeq(k, \ell) \Leftrightarrow(i, j+1) \succeq(k, \ell+1)$.

In the remainder of the paper, we use the ordering $\succeq$ rather than $R$. In the presence of dominance and conditional independence, this involves no loss of generality; see Theorem 2.

Clearly, the case $n=1$ is degenerate. If $n=2$, monotonicity determines a unique ordering. Furthermore, parallel-displacement invariance is implied by monotonicity if 
$n=3$. Therefore, from now on, we assume that $n$ is greater than or equal to four, in which case the two axioms are independent.

An ordering $\succeq$ on $\mathcal{S}[n]$ can be represented diagrammatically in a convenient manner. For a pair of distinct points $(i, j),(k, \ell) \in \mathcal{S}[n]$, an arrow from $(i, j)$ to $(k, \ell)$ indicates that $(i, j) \succeq(k, \ell)$. Consequently, if there is an arrow pointing from $(i, j)$ to $(k, \ell)$ only (and no arrow pointing from $(k, \ell)$ to $(i, j))$, we say that there is a single-arrow from $(i, j)$ to $(k, \ell)$ corresponding to $(i, j) \succ(k, \ell)$. If there is an arrow pointing from $(i, j)$ to $(k, \ell)$ and an arrow pointing from $(k, \ell)$ to $(i, j)$, in which case we speak of a double-arrow between these two points, we have $(i, j) \sim(k, \ell)$. Clearly, monotonicity implies that all horizontal arrows are single-arrows pointing right, all vertical arrows are single-arrows pointing up and all arrows with positive slope are single-arrows pointing north-east. Loosely speaking (we will be more precise below), parallel-displacement invariance requires that all parallel single-arrows point in the same direction and if there exists a double-arrow, then all arrows parallel to it are double-arrows. As an example, suppose that $n=4$. Figure 1 illustrates the ordering $\succeq$ on $\mathcal{S}[4]$ defined by

$$
(1,1) \succ(1,2) \succ(2,2) \succ(1,3) \succ(2,3) \succ(1,4) \succ(3,3) \succ(2,4) \succ(3,4) \succ(4,4)
$$

(all other arrows are determined by transitivity). Clearly, this ordering satisfies monotonicity and parallel-displacement invariance.

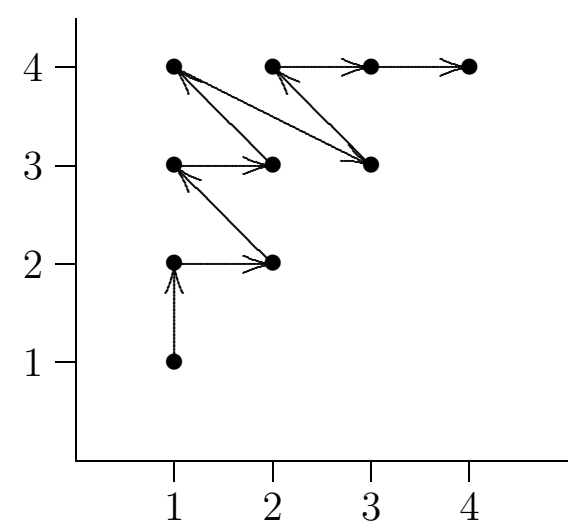

Figure 1: An ordering on $\mathcal{S}[4]$.

We now provide a diagrammatic description of all orderings $\succeq$ on $\mathcal{S}[n]$ satisfying monotonicity and parallel-displacement invariance. Given Theorem 2, this also characterizes the class of all decision rules under complete uncertainty satisfying dominance, conditional 
independence and translation neutrality. The idea is to use monotonicity and paralleldisplacement invariance to reduce the information needed to identify an ordering on $\mathcal{S}[n]$. We know that all positively-sloped arrows are single-arrows pointing north-east as an immediate consequence of monotonicity. Therefore, it is sufficient to include negativelysloped, vertical and horizontal arrows (the direction of vertical and horizontal arrows is determined by monotonicity as well but we include them in order to obtain a more convenient representation; the reason for this choice will become clear later on). Because parallel-displacement invariance implies that all parallel arrows point in the same direction, we can without loss of generality restrict attention to arrows that connect points in $\mathcal{S}[n]$ with $(1, n)$ and, for each slope, have maximal length. To characterize the resulting geometrical figure, we need the following definition.

A quiver in $\mathcal{S}[n]$ is a collection of negatively-sloped, vertical and horizontal arrows of maximal length for each slope connecting points in $\mathcal{S}[n]$ with $(1, n)$ such that:

1. the vertical arrow is a single-arrow that points up;

2. the horizontal arrow is a single-arrow that points right;

3. if a single-arrow or a double-arrow points south-east, then any negatively-sloped arrow whose slope has a smaller absolute value is a single-arrow that points southeast.

The last property in the definition of a quiver implies that if an arrow (single or double) points north-west, then any negatively-sloped arrow whose slope has a larger absolute value is a single-arrow that points north-west. Clearly, there can be no more than one double-arrow in any quiver.

Any quiver in $\mathcal{S}[n]$ uniquely determines an ordering $\succeq$ on $\mathcal{S}[n]$ satisfying monotonicity and parallel-displacement invariance in the following way. We draw all horizontal arrows as single-arrows pointing right, all vertical arrows as single-arrows pointing up and all positively-sloped arrows as single-arrows pointing north-east. Finally, we choose the direction of any negatively-sloped arrow in accordance with the parallel negatively-sloped arrow in the quiver. It is clear that the so-defined ordering $\succeq$ satisfies monotonicity and parallel-displacement invariance. As we show in the following theorem, the reverse implication is also true: any ordering on $\mathcal{S}[n]$ satisfying the two axioms must be determined by a quiver. We therefore obtain the following characterization result. As mentioned above, we assume that $n \geq 4$ to rule out degenerate cases and to ensure that the axioms are independent. 
Theorem 3. Suppose $n \geq 4$. An ordering $\succeq$ on $\mathcal{S}[n]$ satisfies monotonicity and paralleldisplacement invariance if and only if $\succeq$ is determined by a quiver.

Proof. It is straightforward to verify that any ordering determined by a quiver satisfies the axioms. Suppose $\succeq$ is monotonic and satisfies parallel-displacement invariance. Monotonicity and transitivity imply that all horizontal arrows must be single-arrows pointing right, all vertical arrows must be single-arrows pointing up, and all positively-sloped arrows must be single-arrows pointing north-east. Moreover, as an immediate consequence of parallel-displacement invariance, any negatively-sloped arrow points south-east (northwest) if and only if all arrows that are parallel to it point in the same direction. To complete the proof that $\succeq$ is determined by a quiver, it remains to show that, if a singlearrow or a double-arrow points south-east, then any negatively-sloped arrow whose slope has a smaller absolute value is a single-arrow that points south-east.

By way of contradiction, suppose there exists a negatively-sloped arrow (single or double) that points south-east and a negatively-sloped arrow (single or double), whose slope has a smaller absolute value, that points north-west. Because all parallel arrows must point in the same direction, we can without loss of generality assume that each of the two arrows connects $(1, n)$ with another element of $\mathcal{S}[n]$. Suppose that the first arrow connects $(1, n)$ and $(k, l)$ and the second arrow connects $(i, j)$ and $(1, n)$. So we have $(i, j) \succeq(1, n)$ and $(1, n) \succeq(k, \ell)$ where, by monotonicity, $i, j, k, \ell \in[n] \backslash\{1, n\}$. Furthermore, we assume that the angle enclosed by these two arrows is maximal among all the pairs of arrows with the stated properties. By transitivity, we obtain

$$
(i, j) \succeq(k, \ell)
$$

Since the slope of the second arrow is smaller in absolute value, we have $i>k$ or $j>\ell$. There are three possible cases.

Case 1. $i \geq k$ and $j \geq \ell$ with at least one strict inequality. In this case, monotonicity implies $(k, \ell) \succ(i, j)$, a contradiction to (4). See Figure 2 for an illustration. 


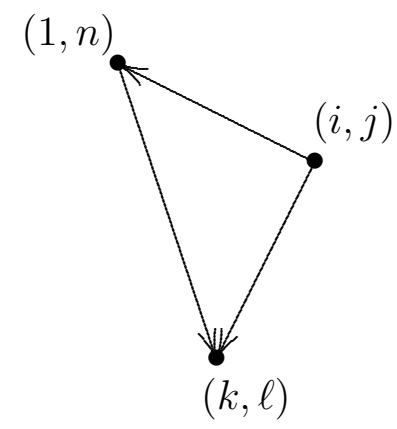

Figure 2: Proof of Theorem 3, case 1.

Case 2. $i<k$ and $j>\ell$. In this case, (4) and parallel-displacement invariance together imply that $(1, n) \succeq\left(i^{\prime}, j^{\prime}\right)$, where $\left(i^{\prime}, j^{\prime}\right)=(k+1-i, \ell+n-j)$. The absolute value of the slope of the arrow $(1, n) \rightarrow\left(i^{\prime}, j^{\prime}\right)$ is larger than that of $(1, n) \rightarrow(k, \ell)$ since it is equal to the absolute value of the slope of $(i, j) \rightarrow(k, l)$. But, as illustrated in Figure 3, this contradicts our assumption that the angle enclosed by the original two arrows is maximal. Indeed, the angle between the arrows $(i, j) \rightarrow(1, n)$ and $(1, n) \rightarrow\left(i^{\prime}, j^{\prime}\right)$ is larger than the angle between the arrows $(i, j) \rightarrow(1, n)$ and $(1, n) \rightarrow(k, \ell)$.

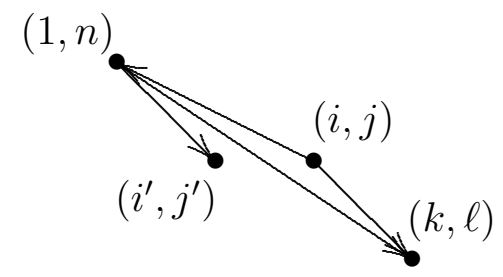

Figure 3: Proof of Theorem 3, case 2.

Case 3. $i>k$ and $j<\ell$. In this case, (4) and parallel-displacement invariance together imply that $\left(i^{\prime}, j^{\prime}\right) \succeq(1, n)$, where $\left(i^{\prime}, j^{\prime}\right)=(i+1-k, j+n-\ell)$. But the existence of this arrow contradicts the maximality of the angle enclosed by the original two arrows in a way similar to Case 2. See Figure 4.

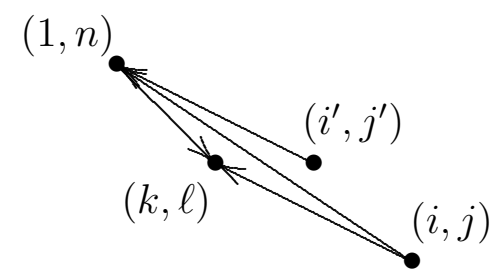

Figure 4: Proof of Theorem 3, case 3.

This exhausts all possibilities and the proof is complete. 


\section{Relative uncertainty aversion}

Theorem 3 suggests a natural way to rank the orderings satisfying our axioms with respect to their degree of uncertainty aversion. For a given negative slope, a single-arrow pointing north-west can be interpreted as representing a more uncertainty averse attitude than a single-arrow with the same slope pointing south-east, and a double-arrow with that slope represents an intermediate attitude towards uncertainty. As an illustration, consider, for example, a quiver in $\mathcal{S}[4]$ and the relative ranking of $(1,4)$ and $(2,3)$. Clearly, if $(2,3)$ is considered better than $(1,4)$, this reflects a higher degree of uncertainty aversion than a ranking that declares $(1,4)$ better than $(2,3)$ because, in the first case, an agent with that preference is willing to forgo the possibility of receiving the best alternative 1 rather than 2 in exchange for being guaranteed the alternative 3 at worst as compared to the worse alternative 4 . Indifference corresponds to an attitude towards uncertainty that is intermediate compared to the two others. A quiver (and, therefore, any of the orderings characterized in Theorem 3) has the convenient property that there is at most one switch from arrows pointing north-west to arrows pointing south-east.

To each arrow connecting $(1, n)$ and $(i, j)$ in a given quiver in $\mathcal{S}[n]$, we attach the fraction $(n-j) /(n-j+i-1)$, expressed in the lowest possible terms. The possible fractions that can be assigned to the arrows in a quiver are those in the set $\mathbf{F}[n-1]$ of elements in a Farey sequence. This set consists of all fractions, expressed in the lowest terms, between zero and one whose denominators do not exceed $n-1$ (see, for instance, Dickson, 1971, or Hardy and Wright, 1960). For example, the set $\mathbf{F}[6]$ is given by

$$
\mathbf{F}[6]=\left\{\frac{0}{1}, \frac{1}{6}, \frac{1}{5}, \frac{1}{4}, \frac{1}{3}, \frac{2}{5}, \frac{1}{2}, \frac{3}{5}, \frac{2}{3}, \frac{3}{4}, \frac{4}{5}, \frac{5}{6}, \frac{1}{1}\right\} .
$$

It is easy to see that the set of fractions assigned to the arrows of a quiver in $\mathcal{S}[7]$ is $\mathbf{F}[6]$.

By definition, a quiver can contain at most one slope to which a double-arrow is assigned. If there exists a double-arrow in a quiver, this quiver is uniquely identified by the Farey fraction associated with the slope of this double-arrow. Each quiver that does not contain a double-arrow can be identified uniquely by a point in the open interval between the largest Farey fraction associated with a single-arrow that points south-east and the smallest Farey fraction associated with a single-arrow that points north-west. For convenience, we choose the mid-point of the interval for this representation. Let $\mathbf{F}^{1 / 2}[n-1]$ be the set of all arithmetic means of any two consecutive elements in $\mathbf{F}[n-1]$ and $\Gamma[n]=\mathbf{F}[n-1] \cup \mathbf{F}^{1 / 2}[n-1] \backslash\{0,1\}$. Any quiver now is characterized by a parameter $\gamma \in \Gamma[n]$. We denote the corresponding ordering on $\mathcal{S}[n]$ by $\succeq_{\gamma}$. As an example, Figure 5 
illustrates the quiver of the ordering $\succeq_{5 / 12}$ in $\mathcal{S}[5]$ (note that $5 / 12$ is the mid-point of the interval $(1 / 3,1 / 2))$.

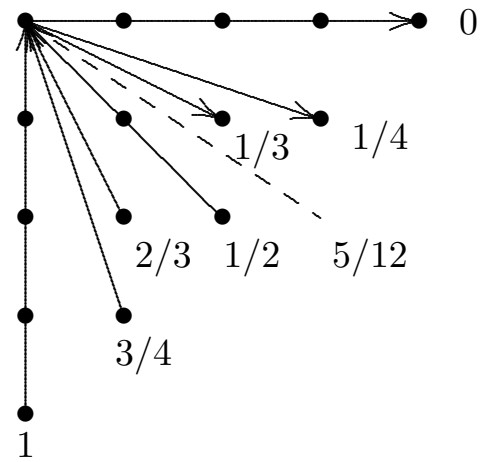

Figure 5: The quiver of the ordering $\succeq_{5 / 12}$ on $\mathcal{S}[5]$.

We can now rank all of the orderings characterized in Theorem 3 with respect to the degree of uncertainty aversion they represent. Given the assignment of Farey fractions to the slopes in a quiver, the lower the value of $\gamma$ at which the switch occurs, the more uncertainty averse is the ordering. Thus, a natural way of classifying these orderings is to declare an ordering $\succeq_{\gamma}$ to be more uncertainty averse than an ordering $\succeq_{\gamma^{\prime}}$ if and only if $\gamma<\gamma^{\prime}$. That is, the parameter $\gamma$ is a very natural indicator of relative uncertainty appeal. As an illustration, consider the case $n=4$. We have

$$
\Gamma[4]=\mathbf{F}[3] \cup \mathbf{F}^{1 / 2}[3] \backslash\{0,1\}=\left\{\frac{1}{6}, \frac{1}{3}, \frac{5}{12}, \frac{1}{2}, \frac{7}{12}, \frac{2}{3}, \frac{5}{6}\right\} .
$$

According to all of the orderings $\succeq_{\gamma}$ for $\gamma \in \Gamma[4],(1,1)$ is best, $(1,2)$ is second-best, $(3,4)$ is second-worst and $(4,4)$ is worst. The remaining rankings are given, in order of decreasing uncertainty aversion, by

$$
\begin{gathered}
(2,2) \succ_{1 / 6}(1,3) \succ_{1 / 6}(2,3) \succ_{1 / 6}(3,3) \succ_{1 / 6}(1,4) \succ_{1 / 6}(2,4) ; \\
(2,2) \succ_{1 / 3}(1,3) \succ_{1 / 3}(2,3) \succ_{1 / 3}(3,3) \sim_{1 / 3}(1,4) \succ_{1 / 3}(2,4) ; \\
(2,2) \succ_{5 / 12}(1,3) \succ_{5 / 12}(2,3) \succ_{5 / 12}(1,4) \succ_{5 / 12}(3,3) \succ_{5 / 12}(2,4) ; \\
(2,2) \sim_{1 / 2}(1,3) \succ_{1 / 2}(2,3) \sim_{1 / 2}(1,4) \succ_{1 / 2}(3,3) \sim_{1 / 2}(2,4) ; \\
(1,3) \succ_{7 / 12}(2,2) \succ_{7 / 12}(1,4) \succ_{7 / 12}(2,3) \succ_{7 / 12}(2,4) \succ_{7 / 12}(3,3) ; \\
(1,3) \succ_{2 / 3}(2,2) \sim_{2 / 3}(1,4) \succ_{2 / 3}(2,3) \succ_{2 / 3}(2,4) \succ_{2 / 3}(3,3) ; \\
(1,3) \succ_{5 / 6}(1,4) \succ_{5 / 6}(2,2) \succ_{5 / 6}(2,3) \succ_{5 / 6}(2,4) \succ_{5 / 6}(3,3) .
\end{gathered}
$$


Due to the decreasing degree of uncertainty aversion as measured by $\gamma$, the pair $(1,4)$ moves up in the ranking as $\gamma$ increases. See Figure 6 for a graphical illustration.
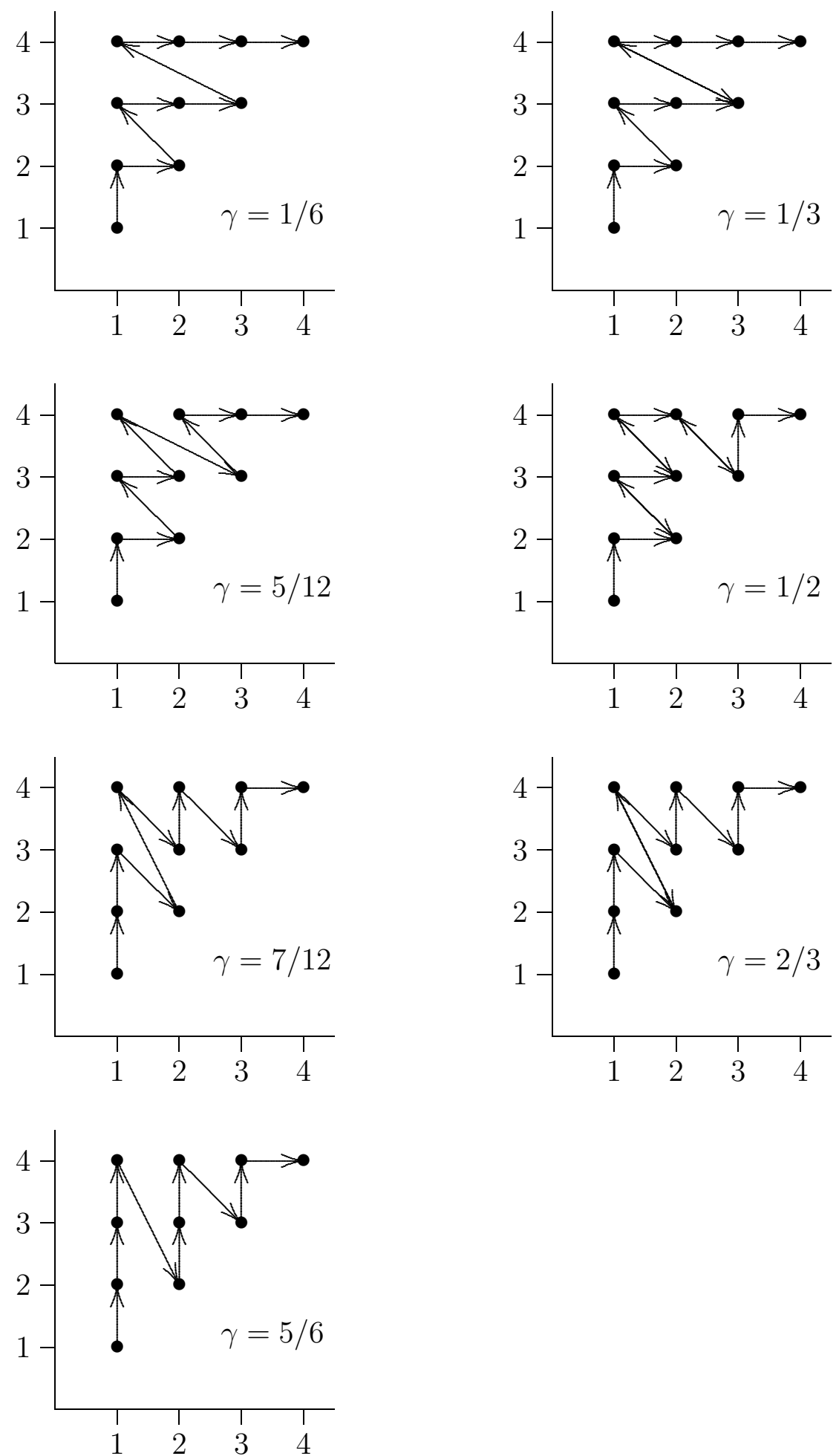

Figure 6: Orderings on $\mathcal{S}[4]$ which satisfy the conditions of Theorem 3. 
The parameterization in terms of Farey fractions also allows us to address an interesting combinatorial issue. The number of orderings satisfying our axioms is given by the number of possible values the parameter $\gamma$ can assume. As is straightforward to verify, this number is given by $|\Gamma[n]|=2|\mathbf{F}[n-1]|-3$.

We can invoke some number-theoretic concepts and known results regarding sets of Farey fractions in analyzing the cardinality of the set $\Gamma[n]$. The Euler function $\phi: \mathbb{N} \rightarrow \mathbb{N}$ is defined by letting $\phi(1)=1$ and, for all $m \geq 2$, by letting $\phi(m)$ be the number of positive integers not exceeding $m$ and relatively prime to $m$. It is easy to see that, in moving from $\mathbf{F}[m]$ to $\mathbf{F}[m+1]$, there are $\phi(m+1)$ new Farey fractions added. Therefore, for all $m \in \mathbb{N}$, the cardinality of $\mathbf{F}[m]$ is $\Phi(m)+1$, where $\Phi(m)=\sum_{h=1}^{m} \phi(h)$. The sets composed of the elements of Farey sequences have been studied extensively and, although there is no explicit formula to calculate the cardinality of $\mathbf{F}[n-1]$ for arbitrary $n$, an asymptotic result is available.

Theorem 4. Asymptotically, $|\Gamma[n]|=6 n^{2} / \pi^{2}+O(n \log n)$.

Proof. Attributing this result to Mertens, Hardy and Wright (1960, p. 268, p. 272) establish in Theorem 330 that, asymptotically, $\Phi(m)=3 m^{2} / \pi^{2}+O(m \log m)$. The conclusion of Theorem 4 now follows immediately from the observation that $|\Gamma[n]|=2|\mathbf{F}[n-1]|-3$ and the fact that $|\mathbf{F}[m]|=\Phi(m)+1$ for all $m \in \mathbb{N}$.

\section{Additive representability}

An interesting property of an ordering of sets is additive representability (see, for example, Fishburn, 1970, Chapter 4). The standard concept of additive representability for the ordering $R$ on $\mathcal{P}[n]$, stipulates the existence of a function $U:[n] \rightarrow \mathbb{R}$ such that the ranking of any two sets $A$ and $B$ is determined by comparing the sums $\sum_{i=1}^{|A|} U\left(a_{i}\right)$ and $\sum_{i=1}^{|B|} U\left(b_{i}\right)$ representing the total utilities of these sets. In view of Theorem 1 , it is clear that this axiom is not compatible with dominance and conditional independence if $n \geq 3$. To see this, suppose $U$ is such a function. By Theorem 1, we must have $U(1)+U(2)+U(3)=U(1)+U(3)$ and thus $U(2)=0$. But dominance requires $U(1)>$ $U(1)+U(2)$, a contradiction. However, Theorem 1 suggests another concept of additive representability presented below.

Additive representability for orderings on $\mathcal{P}[n]$. There exists a function $U:[n] \rightarrow \mathbb{R}$ such that, for all $A, B \in \mathcal{P}[n]$,

$$
A R B \Leftrightarrow U\left(a_{1}\right)+U\left(a_{|A|}\right) \geq U\left(b_{1}\right)+U\left(b_{|B|}\right) .
$$


Unlike in the classical case, the total utilities of the two extremes (the best and the worst elements) are compared here, not the total utilities of the sets. This translates into the following property of an ordering on $\mathcal{S}[n]$.

Additive representability for orderings on $\mathcal{S}[n]$. There exists a function $U:[n] \rightarrow \mathbb{R}$ such that, for all $(i, j),(k, \ell) \in \mathcal{S}[n]$,

$$
(i, j) \succeq(k, \ell) \Leftrightarrow U(i)+U(j) \geq U(k)+U(\ell)
$$

We focus on the latter axiom and we prove that additive representability is guaranteed for all orderings $\succeq_{\gamma}$ with $\gamma \in \mathbf{F}^{1 / 2}[n-1]$, that is, for those orderings satisfying monotonicity and parallel-displacement invariance that are antisymmetric.

Theorem 5. Suppose $n \geq 4$. If $\gamma \in \mathbf{F}^{1 / 2}[n]$, then $\succeq_{\gamma}$ satisfies additive representability.

Proof. Let $N=n(n+1) / 2$ be the number of elements in $\mathcal{S}[n]$ and suppose $\gamma \in \mathbf{F}^{1 / 2}[n]$ is such that

$$
\left(i_{1}, j_{1}\right) \succ_{\gamma}\left(i_{2}, j_{2}\right) \succ_{\gamma} \ldots \succ_{\gamma}\left(i_{N}, j_{N}\right)
$$

To each $t \in[N-1]$, we assign a linear function $f_{t}: \mathbb{R}^{n} \rightarrow \mathbb{R}$ such that, for all $\mathbf{x} \in \mathbb{R}^{n}$,

$$
f_{t}(\mathbf{x})=x_{i_{t}}+x_{j_{t}}
$$

If the order (5) is additively representable, then the following system of $N-1$ linear inequalities

$$
\begin{aligned}
f_{1}(\mathbf{x})-f_{2}(\mathbf{x}) & >0 \\
f_{2}(\mathbf{x})-f_{3}(\mathbf{x}) & >0 \\
& \vdots \\
f_{N-1}(\mathbf{x})-f_{N}(\mathbf{x}) & >0
\end{aligned}
$$

is consistent with $x_{i}=U(i)$, where $U$ is the corresponding utility function. The reverse is also true, hence this system is consistent if and only if $\succeq_{\gamma}$ is additively representable.

To show that the system is consistent, note first that the $t^{\text {th }}$ inequality

$$
f_{t}(\mathbf{x})-f_{t+1}(\mathbf{x})>0
$$

defines an open half-space $H_{t}$ in $\mathbb{R}^{n}$ determined by the corresponding hyperplane $f_{t}(\mathbf{x})-$ $f_{t+1}(\mathbf{x})=0$. For each $t \in[N-1]$, we define the vector

$$
\mathbf{v}_{t}=\mathbf{e}_{i_{t}}+\mathbf{e}_{j_{t}}-\mathbf{e}_{i_{t+1}}-\mathbf{e}_{j_{t+1}}
$$


where, for all $i \in[n], \mathbf{e}_{i}=(0, \ldots, 1, \ldots, 0)^{T}$ is the $i^{t h}$ unit vector in the standard basis of $\mathbb{R}^{n}$. The vector $\mathbf{v}_{t}$ is an inner normal vector of $H_{t}$, that is, $\mathbf{x} \in H_{t}$ if and only if the dot product of $\mathbf{v}_{t}$ and $\mathbf{x}$ is positive. The inconsistency of the system above is equivalent to the existence of a non-trivial linear combination

$$
a_{1} \mathbf{v}_{1}+a_{2} \mathbf{v}_{2}+\cdots+a_{N-1} \mathbf{v}_{N-1}=\mathbf{0}
$$

of $\mathbf{v}_{1}, \ldots, \mathbf{v}_{N-1}$ with non-negative coefficients $a_{1}, \ldots, a_{N-1}$ (see, for example, Theorem 2.9 in Gale, 1960, p. 48). Because the coordinates of $\mathbf{v}_{1}, \ldots, \mathbf{v}_{N-1}$ are integers, the coefficients of this linear combination can be chosen to be integers as well.

Now we start compiling a system of linear inequalities in the following way. For each $k \in[N-1]$ we include the inequality $\left(i_{1}, j_{1}\right) \succ_{\gamma}\left(i_{2}, j_{2}\right)$, corresponding to vector $\mathbf{v}_{k}$, exactly $a_{k}$ times. Some of them will be listed several times, some (for which the corresponding coefficient of $\mathbf{v}_{k}$ is zero) will not be listed at all. We obtain a system of comparisons

$$
\begin{aligned}
& a_{1}\left\{\begin{array}{ccc}
\left(i_{1}, j_{1}\right) & \succ_{\gamma} & \left(i_{2}, j_{2}\right) \\
& \vdots \\
\left(i_{1}, j_{1}\right) & \succ_{\gamma} & \left(i_{2}, j_{2}\right)
\end{array}\right. \\
& a_{N-1}\left\{\begin{array}{ccc}
\left(i_{N-1}, j_{N-1}\right) & \succ_{\gamma} & \left(i_{N}, j_{N}\right) \\
& \vdots \\
\left(i_{N-1}, j_{N-1}\right) & \succ_{\gamma} & \left(i_{N}, j_{N}\right)
\end{array}\right.
\end{aligned}
$$

The characteristic feature of this sytem is that, for each integer $t \in[n]$, the number of times $t$ occurs on the left side of the system is the same as the number of times it occurs on the right side. This follows from the fact that all positive occurrences of $\mathbf{e}_{t}$ in (6) are cancelled by the negative occurrences of $\mathbf{e}_{t}$.

Since, by assumption, $\gamma$ is such that $\succ_{\gamma}$ is antisymmetric, we have either $\gamma>1 / 2$ or $\gamma<1 / 2$. In the former case, we have $i_{t}+j_{t}>i_{t+1}+j_{t+1}$ for all $t \in[N-1]$ and in the latter, it follows that $i_{t}+j_{t}<i_{t+1}+j_{t+1}$ for all $t \in[N-1]$. Both possibilities contradict the property of the above system of comparisons that the number of times an element of $[n]$ occurs is the same on both sides.

Scott (1964) shows that for the classical additive-representability property to hold, it is necessary and sufficient that the so-called cancellation conditions are satisfied. For our version of additive representability, an analogous set of cancellation conditions can be formulated. We do not present these conditions explicitly but the reader familiar with the 
aforementioned paper by Scott will notice that the previous proof proceeds by showing that these cancellation conditions are satisfied by any antisymmetric ordering on $\mathcal{S}[n]$.

For $n \leq 5$, additive representability is satisfied even for orderings that allow for indifference, that is, for all orderings $\succeq_{\gamma}$ with $\gamma \in \Gamma[n]$. This is no longer the case if $n \geq 6$. For example, if $n=6$, the ordering $\succeq_{2 / 3}$ is not additively representable. By way of contradiction, suppose that $\succeq_{2 / 3}$ is additively representable. For notational convenience, let $x_{i}=U(i)$ for all $i \in[6]$. Since $(1,4) \sim_{2 / 3}(2,2),(2,5) \sim_{2 / 3}(3,3),(3,6) \sim_{2 / 3}(4,4),(1,5) \sim_{2 / 3}(2,3)$, $(2,6) \sim_{2 / 3}(3,4)$, and $(1,6) \sim_{2 / 3}(2,4)$, we have $x_{1}+x_{4}=2 x_{2}, x_{2}+x_{5}=2 x_{3}, x_{3}+x_{6}=2 x_{4}$, $x_{1}+x_{5}=x_{2}+x_{3}, x_{2}+x_{6}=x_{3}+x_{4}$, and $x_{1}+x_{6}=x_{2}+x_{4}$. Thus, $\mathbf{x}=\left(x_{1}, \ldots, x_{6}\right)^{T}$ must satisfy $M \mathbf{x}=\mathbf{0}$, where

$$
\mathbf{M}=\left(\begin{array}{rrrrrr}
1 & -2 & 0 & 1 & 0 & 0 \\
0 & 1 & -2 & 0 & 1 & 0 \\
0 & 0 & 1 & -2 & 0 & 1 \\
1 & -1 & -1 & 0 & 1 & 0 \\
0 & 1 & -1 & -1 & 0 & 1 \\
1 & -1 & 0 & -1 & 0 & 1
\end{array}\right)
$$

However, any solution $\mathbf{x}$ to this system of equations is such that $x_{i}=x_{j}$ for all $i, j \in[6]$ as its null space is spanned by the vector $(1,1,1,1,1,1)^{T}$. But dominance requires $x_{i}>x_{i+1}$ for all $i \in[5]$, a contradiction.

\section{Concluding remarks}

This paper provides an intuitively appealing notion of uncertainty aversion in set-based models of choice under uncertainty. It turns out that an unambiguous ranking of decision rules with respect to their relative uncertainty aversion can be established for a large class of rules characterized by a small set of plausible axioms. A new concept of additive representability of orderings is defined and it is shown that all antisymmetric orderings in our class are additively representable.

An interesting further direction of research would be to explore the notion of relative uncertainty aversion in models where the underlying relation on the set of objects $X$ has different properties. Implicitly, our definition of relative uncertainty aversion is distancebased with the simplest distance $d(i, j)=|i-j|$ on $X=[n]$ being employed, which reflects the assumption that the underlying preference relation on $X$ is an antisymmetric ordering. One important alternative is obtained when the underlying preference relation on $X$ is 
single-peaked. Another interesting case arises if it is assumed that the set $X$ consists of the vertices of the $k$-dimensional unit cube and to employ the Hamming distance. An analysis of relative uncertainty aversion in this setting would clarify some important behavioral aspects of the fallback-bargaining procedure studied by Brams, Kilgour and Sanver (2003). 


\section{References}

Arrow, K.J. (1971), Essays in the theory of risk-bearing, Markham, Chicago.

ArRow, K.J. AND L. HuRwiCz (1972), "An optimality criterion for decision-making under ignorance," in: C.F. Carter and J.L. Ford (eds.), Uncertainty and Expectations in Economics: Essays in Honour of G.L.S. Shackle, Basil Blackwell, Oxford, 1-11.

Bandyopadhyay, T. (1988), "Extensions of an order on a set to the power set: some further observations," Mathematical Social Sciences 15, 81-85.

Barberà, S., C.R. Barrett and P.K. Pattanaik (1984), "On some axioms for ranking sets of alternatives," Journal of Economic Theory 33, 301-308.

Barberà, S., W. Bossert And P.K. Pattanaik (2004), "Ranking sets of objects," in: S. Barberà, P. Hammond and C. Seidl (eds.), Handbook of Utility Theory, Vol. 2: Extensions, Kluwer, Dordrecht, 893-977.

BarberÀ, S. And P.K. Pattanaik (1984), "Extending an order on a set to the power set: some remarks on Kannai and Peleg's approach," Journal of Economic Theory 32, $185-191$.

Barrett, C.R. And P.K. Pattanaik (1993), "Decision-making under complete uncertainty," in: S. Sen (ed.), Uncertainty and Risk in Economic Life, Edward Elgar, Aldershof, $20-38$.

Bossert, W. (1989), "On the extension of preferences over a set to the power set: an axiomatic characterization of a quasi-ordering," Journal of Economic Theory 49, 84-92.

Bossert, W. (1997), "Uncertainty aversion in nonprobabilistic decision models," Mathematical Social Sciences 34, 191-203.

Bossert, W., P.K. Pattanaik and Y. Xu (2000), "Choice under complete uncertainty: axiomatic characterizations of some decision rules," Economic Theory 16, 295312 .

Brams, S.J, D.M. Kilgour, And M.R. Sanver (2003), "A minimax procedure for negotiating multilateral treaties," Working Paper, New York University, Department of Politics.

Chew, S.H., E. Karni And Z. SAFra (1987), "Risk aversion in the theory of expected utility with rank-dependent probabilities," Journal of Economic Theory 42, 370-381. 
Cohen, M. AND J.Y. JAFFRAY (1980), "Rational behavior under complete ignorance," Econometrica 48, 1281-1299.

Dickson, L.E. (1971), History of the Theory of Numbers, Chelsea, New York.

Epstein, L.G. (1999), "A definition of uncertainty aversion," Review of Economic Studies 66, 579-608.

Fishburn, P.C. (1970), Utility Theory for Decision Making, Wiley, New York.

Fishburn, P.C. (1984), "Comment on the Kannai-Peleg impossibility theorem for extending orders," Journal of Economic Theory 32, 176-179.

Gale, D. (1960), The Theory of Linear Economic Models, McGraw-Hill, New York.

GÄRDEnFors, P. (1976), "Manipulation of social choice functions," Journal of Economic Theory 13, 217-228.

Hardy, G.H. And E.M. Wright (1960), An Introduction to the Theory of Numbers, Oxford University Press, Oxford.

Heiner, R.A. And D.J. PACKARD (1984), "A uniqueness result for extending orders; with applications to collective choice as inconsistency resolution," Journal of Economic Theory 32, 180-184.

Holzman, R. (1984a), "An extension of Fishburn's theorem on extending orders," Journal of Economic Theory 32, 192-196.

Holzman, R. (1984b), "A note on the redundancy of an axiom in the Pattanaik-Peleg characterization of the lexicographic maximin extension," Social Choice and Welfare 1, $123-125$.

Kannai, Y. And B. Peleg (1984), "A note on the extension of an order on a set to the power set," Journal of Economic Theory 32, 172-175.

Kim, K.H. And F.R. Roush (1980), "Preferences on subsets," Journal of Mathematical Psychology 21, 279-282.

Luce, R.D. And H. Raiffa (1957), Games and Decisions, Wiley, New York.

MASKIn, E. (1979), "Decision-making under ignorance with implications for social choice," Theory and Decision 11, 319-337.

Milnor, J. (1954), "Games against nature," in: R. Thrall, C. Coombs and R. Davis (eds.), Decision Processes, Wiley, New York, 49-59. 
Nehring, K. And C. Puppe (1996), "Continuous extensions of an order on a set to the power set," Journal of Economic Theory 68, 456-479.

Nitzan, S. And P.K. Pattanaik (1984), "Median-based extensions of an ordering over a set to the power set: an axiomatic characterization," Journal of Economic Theory 34, $252-261$.

Pattanaik, P.K. And B. Peleg (1984), "An axiomatic characterization of the lexicographic maximin extension of an ordering over a set to the power set," Social Choice and Welfare 1, 113-122.

Pratt, J. (1964), "Risk aversion in the small and in the large," Econometrica 32, 122136.

Rawls, J. (1971), A Theory of Justice, Harvard University Press, Cambridge.

Savage, L.J. (1954), The Foundations of Statistics, Wiley, New York.

SCHMEIDLER, D. (1989), "Subjective probability and expected utility without additivity," Econometrica 57, 571-587.

Scotт, D. (1964), "Measurement structures and inequalities," Journal of Mathematical Psychology 1, 233-247.

von Neumann, J. And O. Morgenstern (1944; second ed. 1947), Theory of Games and Economic Behavior, Princeton University Press, Princeton.

WAKkeR, P. (1990), "Characterizing optimism and pessimism directly through comonotonicity," Journal of Economic Theory 52, 453-463. 